\title{
LORA (Long Range) Based Low Power Emergency Tracking System in Disaster Areas
}

\author{
S. Sweetline Shamini ${ }^{\text {a,1 }}$, A.P. Anuja ${ }^{\text {a }}$, B. Archana ${ }^{\text {a }}$, P. Premalakshmi ${ }^{\text {a }}$ \\ ${ }^{a}$ Department of Electronics and Communication Engineering, Sri Sai Ram Institute \\ of Technology, Chennai, Tamil Nadu, India
}

\begin{abstract}
The communication network is one of the major assistances for disaster management purposes. At each disaster phase, the information flow between the disaster management departments and the population is critical, as it destroys the whole communication infrastructure where the base station to Mobile Switching Centre (MSC) connectivity is broken, power generators are exhausted, and the network may suffer from congestion. Even when radio equipment works, police officers, rescue teams, medical teams cannot communicate with each other because of incompatible radios. To analyze the characteristics of the geological environment in the disaster region and to alert the Disaster Management Department, this solution based on LoRa has been proposed. The penultimate goal of this paper is to fasten the rescue operations.
\end{abstract}

Keywords. LoRa, Spreading Factor, Communication, Disaster Management

\section{Introduction}

Often calamities such as earthquakes, hurricanes, floods affect communication services like mobile phone networks, commercial radio networks, and other communication services infrastructures, unavailable for any disaster management activities. LoRa is implemented using the non-cellular Low Power Wide Area Network (LPWAN), a wireless communication network protocol operating over a range of $2 \mathrm{~km}-5 \mathrm{~km}$ without Line of Sight (LoS) and about $15 \mathrm{~km}-20 \mathrm{~km}$ with Line of Sight. Since it works under LPWAN the power consumption varies between $10 \mathrm{~mW}-100 \mathrm{~mW}$ which is minimum when compared to Wi-Fi, LTE, EDGE. LoRa uses a modulation technique which is similar to Chirp spread spectrum modulation, uses unlicensed radio spectrum in the ISM band that provides seamless interoperability between devices. Henceforth this idea is implemented using LoRa that focuses on data transmission which requires a minimum data rate over a wider area. LoRa wireless outcomes are the best alternative for eliminating the need for repeaters, minimizing cost consumption, improving battery life, and also enhancing network capacity. LoRa transceivers are Long Range wireless modem helps in preventing and improving the selectivity over traditional modulation approaches, to overcome the flaws of conventional methodology balancing between longer range of transmission as well as high interference immunity by minimizing current consumption.

\footnotetext{
${ }^{1}$ S. Sweetline Shamini, Department of Electronics and Communication Engineering, Sri Sai Ram Institute of Technology, Chennai, Tamil Nadu, India, Email: sweetlineshamini.ece@sairamit.edu.in.
} 


\section{Existing Methods}

With respect to the paper, Emergency communication in IoT scenarios by means of a transparent LoRaWAN enhancement published by Emiliano Sisinni in 2020, concluded that their proposal is effective in reducing the loss of emergency messages without retries [1]. The LoRa (Long-Range) High-Density Sensors for Internet of Things, published by Alexandru Lavric in 2019, determined the maximum number of sensors that can be integrated to perform IoT service [2]

Post Disaster Relief Operations using Ad-hoc Network, Zigbee protocol evaluates whether peer to peer communication can reach out the long distance without any loss of information [3]. In reference to the proposed paper, Radio Data Infrastructure for Remote Monitoring System using LoRa Technology published by Ravi Kishore Kodali in 2017, concluded that The LoRa Wireless technology offers long range for communication for IoT services [4]. In disaster regions, Walkie Talkies and Citizen Band Radios are used which support communication for a shorter distance where walkie-talkies will perform only 1/10th of its performance and the efficiency of Ham or Amateur radios are also deteriorated at times of poor weather condition [5].

\section{Proposed Solution}

\subsection{Introduction}

Communication is vital for providing the right support at the time of disaster. In this solution, we have used LoRa for long-distance transmission which allows us to make communication way easier and more cost-effective [6].

\subsection{Working}

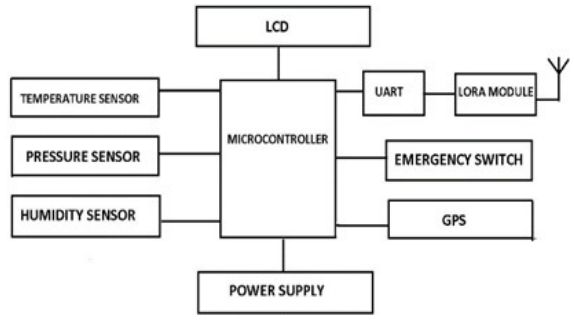

Figure 1. Block Diagram - Transmitter section

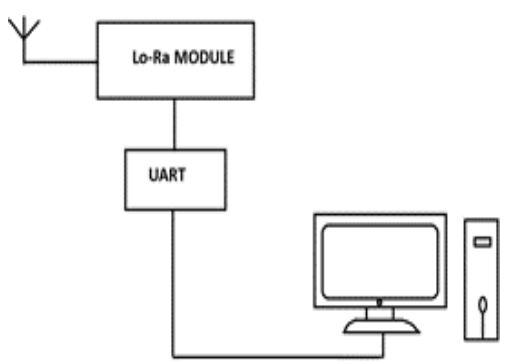

Figure 2. Block Diagram -Receiver section

In Figure 1, Transmitter section and in Figure 2, Receiver section block diagrams are presented. At the time of any natural calamity, when there is no proper infrastructurebased communication, the proposed solution may be used. The emergency switch is pressed physically, the transmitting unit is triggered, that is the microcontroller continuously looks for the emergency impulse and this impulse is applied using a switch. With the press of the emergency switch, the external components are also 
triggered and start reading the values from the surrounding environment. The GPS unit sends the current location and the sensor data such as temperature sensor, humidity, pressure sensor to the microcontroller. The power supply acts as a source for the microcontroller. The type of emergency message is prerecorded in different switches and could be transmitted easily [7].

At the receiver section that is placed at a significant distance, the transmitted data is received by the LoRa module and data are viewed in a tracker application.

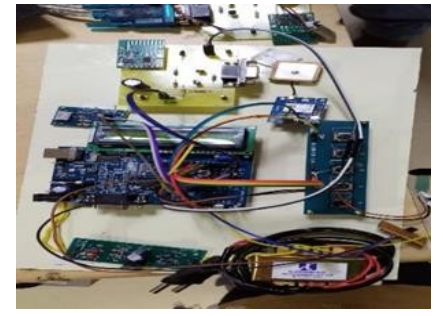

Figure 3. Implementation of transmission

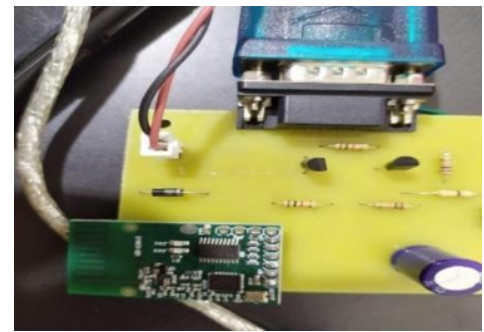

Figure 4. Implementation of receiver section

\subsection{Output}

For the implementations shown in Figure 3 and figure 4, the outputs are taken. An application was built using visual basic code to view the received data via the serial port from the LoRa module. This data can be logged with the help of an application called Tera Term into a CSV file from where we could visualize the data in the form of graphs in Figure 5 and Figure 6 for better understanding of the disaster hit zone.

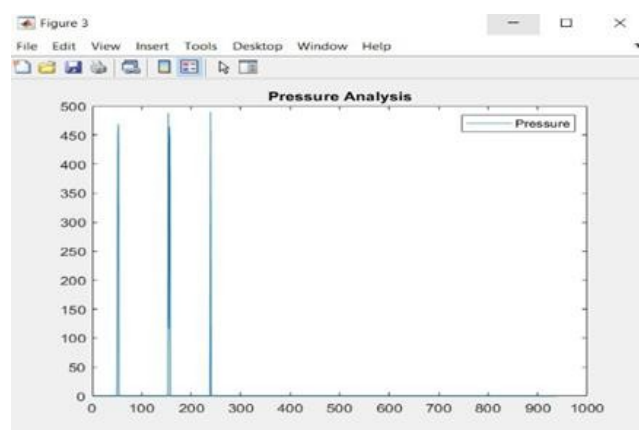

Figure 5. A Plot of received Pressure data.

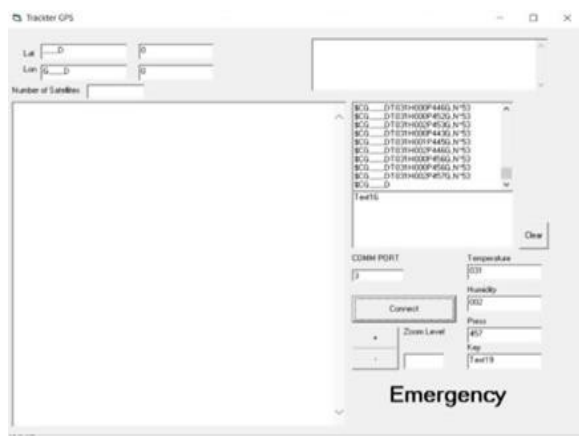

Figure 6. Tracker application showing the received data

LoRa modulation technique uses orthogonal spreading factors i.e. it can send messages concurrently with different spreading factors with the same frequency and can be received without interference as they appear noise to each other [8]. Thus, this technique offers high immunity to multipath fading and interference.

The capacity of LoRa is given by, 
Capacity $=1 *\left(\sum_{i=1}^{12} S F_{i}\right.$ equivalent bit error rate $)$

From Semtech LoRa specification,

Capacity $_{\text {LoRa }}=12.156 \mathrm{~kb} / \mathrm{s}$

The Figure 7, shows the depiction of 500 random messages each of 25 bytes and the nodes/ devices are allowed to select a random spreading factor for concurrent use of the available spectrum. Here, Green depicts efficient transmissions while the Red depicts the failed transmissions with the use of the same spreading factor.

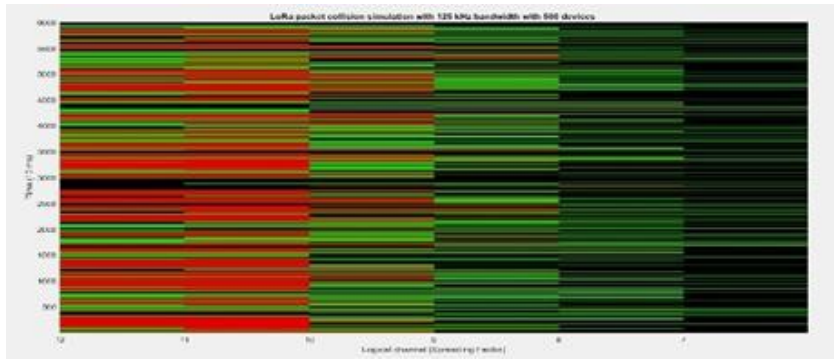

Figure 7. Simulation of collision of packets for different spreading factors



Figure 8. Simulation showing the Packet

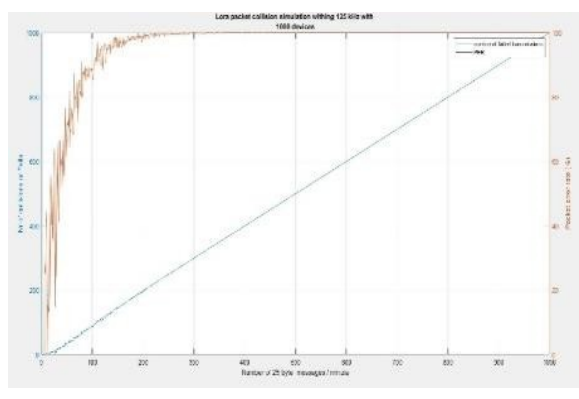

Figure 9. Simulation showing the collision and collision

Figure 8 and Figure 9 shows the simulation outputs. Packet Error Rate with a spreading factor of 12 Error Rate with a spreading factor of 7 . The above figures show a comparison between the ideal spreading factor (SF7) and the worst spreading factor (SF12).

If we consider the ideal case where we have only used a spreading factor of 7 , it results in a short message of about $36 \mathrm{~ms}$. Here the collisions are minimum and the bit rate is maximum while the range of transmission is around $2 \mathrm{~km}$ only.

In another scenario, the spreading factor is fixed as 12 . This results in long messages (around $682 \mathrm{~ms}$ ). Here the collisions are high and the bit rate is reduced but the transmission range is up to $10 \mathrm{~km}$.

LoRa chooses the right spreading factor by considering the distance between the transmitter and the receiver. As this setup consists of several sensors, an optimal distance of $2 \mathrm{~km}$ is chosen as a range of communication to avoid packet loss. 


\section{Performance Analysis}

To examine the performance of the proposed solution, as per the Figure 10, the following test was conducted. A random location was fixed as the transmitter and six random locations at different distances were chosen as receivers. The transmitting section was kept at a height of $5 \mathrm{~m}$ and the sensed data were transmitted to the receiver at each location and this experiment was repeated nine times. The Table 1, shows the success rate for each location.

From the table, we can conclude that up to a distance of $500 \mathrm{~m}$, none of the trials failed. But as the distance gradually increases the success rate decreases. For a distance of $2 \mathrm{~km}$, the success rate was about $70 \%$. This could be improved by placing the transmitting module at a height of $30 \mathrm{~m}$ as it would improve the line of sight, enabling us to establish communication to a greater distance.

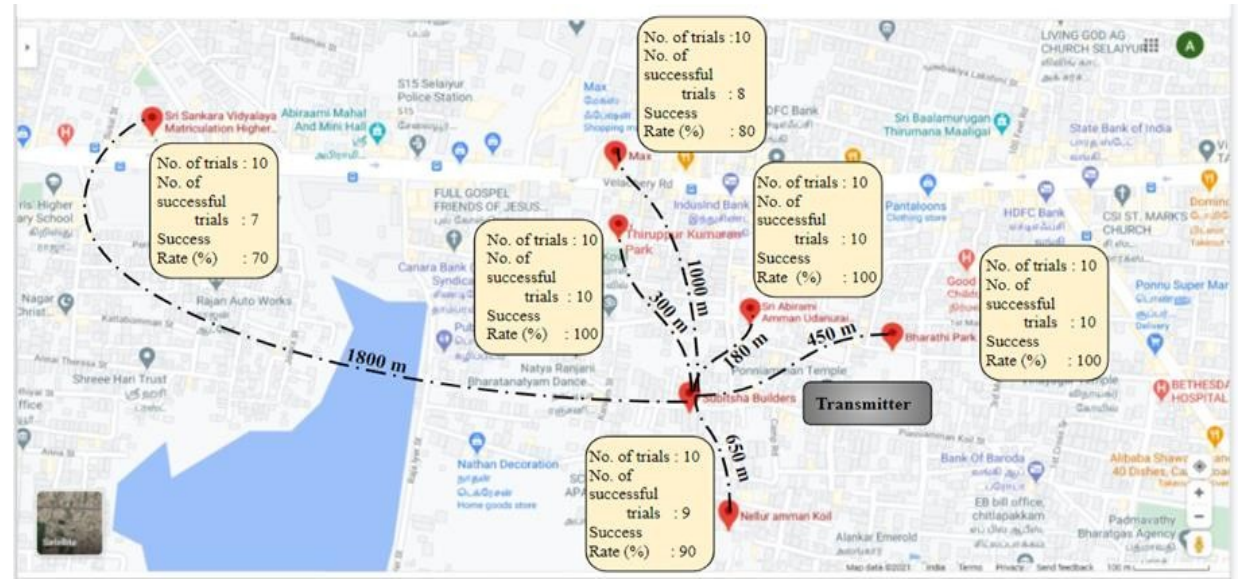

Figure 10. A Track of Performance Analysis

Table 1. Performance Analysis outcomes considering range

No. of Trials : 10

\begin{tabular}{|l|l|l|}
\hline $\begin{array}{l}\text { Distance between the Transmitter and } \\
\text { Receiver } \\
\text { (in m) }\end{array}$ & No. of successful Trails & Success Rate (in \%) \\
\hline 180 & 10 & 100 \\
\hline 300 & 10 & 100 \\
\hline 450 & 10 & 100 \\
\hline 650 & 9 & 90 \\
\hline 1000 & 8 & 80 \\
\hline 1800 & 7 & 70 \\
\hline
\end{tabular}

5.

\section{Conclusion}

As discussed already, calamities are inevitable, and establishing communication during this situation is a key factor to bounce back. Thus, this solution based on LoRa 
provides us a way to control the spreading factor thereby efficiently controlling the range of transmission which was not possible using other protocols like Wi-Fi and also consumes much lesser power. With such an increase in range of the transmission, the need for a proper centralized base station, all the necessary data could be obtained and this module can also be mounted on Unmanned Aerial Vehicles, thus monitoring places affected by disasters such as forest fire, where human being survival is impossible. Hence, this setup would act as an optimal solution with high reliability, extremely low cost, secure and high interoperability.

\section{6. $\quad$ Future Work}

Alongside data transmission, this setup can be extended for voice and image transmission. Since, LoRa is suitable for communication involving low data rates, identifying proper compression techniques for voice and image is required. Doing so will enable us to analyze and understand the environment from a broader perspective and help us to calculate the remedial measures soon and with even more high accuracy.

\section{References}

[1] Kodali, R. K. (2017). Radio data infrastructure for remote monitoring system using LoRa technology. In 2017 International Conference on Advances in Computing, Communications and Informatics, ICACCI 2017 (Vol. 2017-January, pp. 467-472). Institute of Electrical and Electronics Engineers Inc. https://doi.org/10.1109/ICACCI.2017.8125884

[2] Sisinni, E., Carvalho, D. F., \& Ferrari, P. (2020). Emergency Communication in IoT Scenarios by Means of a Transparent LoRaWAN Enhancement. IEEE Internet of Things Journal, 7(10), 1068410694. https://doi.org/10.1109/JIOT.2020.3011262

[3] P.Lavanya, B.Somi, S.Dev Nandan, J.Mani Mohana Krishna, N.Veeraiah, Post Disaster Relief Operations using Ad-hoc Network, 2020

[4] Rene Tendeng, YoungDoo Lee, and Insoo Koo, Implementation and Measurement of Spectrum Sensing for Cognitive Radio Networks Based on LoRa and GNU Radio

[5] Alexandru Lavric, Valentin Popa, Internet of Things and LoRaTM Low-Power Wide Area Networks: A Survey, 2017.

[6] Ganesh Babu R., Karunakaran A., Manikandan G., Kalimuthu Kumar S., Selvameena R. (2021) IoT in Smart Automation and Robotics with Streaming Analytical Challenges. In: Singh K.K., Nayyar A., Tanwar S., Abouhawwash M. (eds) Emergence of Cyber Physical System and IoT in Smart Automation and Robotics. Advances in Science, Technology \& Innovation (IEREK Interdisciplinary Series for Sustainable Development). Springer, Cham. https://doi.org/10.1007/978-3-030-66222-6_7

[7] Markkandan, S., Malarvizhi, C., Raja, L., Kalloor, J., Karthi, J., \& Atla, R. (2021), "Highly compact sized circular microstrip patch antenna with partial ground for biomedical applications", Materials Today: Proceedings. https://doi.org/10.1016/j.matpr.2021.04.480

[8] C. C., G. B. R., S. M., A. M., B. R. and M. Balaji, "Machine Learning Based Condition Recognition System for Bikers," 2020 7th International Conference on Smart Structures and Systems (ICSSS), 2020, pp. 1-6, https://doi.org/10.1109/ICSSS49621.2020.9202245 